\title{
State-to-state rate constants for collision induced energy transfer of electronically excited $\mathrm{NH}_{2}$ with $\mathrm{NH}_{3}$
}

\author{
Jörg Lindner ${ }^{\mathrm{a})}$ \\ Max-Planck-Institut für biophysikalische Chemie, AG biomolekulare und chemische Dynamik (072), \\ Am Fassberg 11, D-37077 Göttingen, Germany \\ Ralf Wilhelm ${ }^{\text {b) }}$ \\ Institut für Quantenoptik, Universität Hannover, Welfengarten 1, D-30167 Hannover, Germany
}

(Received 2 August 2001; accepted 12 June 2002)

Collisional energy transfer of $\mathrm{NH}_{2}$ in its electronically excited state $\widetilde{A}^{2} A_{1}$ is investigated with time-resolved Fourier transform emission spectroscopy. $\mathrm{NH}_{2}$ is produced by photodissociation of $\mathrm{NH}_{3}$ and relaxed to low rotational levels before excitation into the electronically excited state. Originating from collisions with $\mathrm{NH}_{3}$, rate constants for total collisional removal and state-to-state rate constants for rotational energy transfer within $v_{2}=4, K_{a}=1$ with collision induced changes of $\left|\Delta K_{c}\right| \leqslant 3$ are determined. The latter rate constants are fitted with several scaling laws. Among these, those based on the energy corrected sudden approximation work best. An approximate potential curve for the anisotropic part of the interaction potential is derived and verified with cross sections obtained with straight line trajectories. The rotational energy transfer originates primarily from collisions with small impact parameters. The observed rate constants for total collisional removal are in accordance with the collision complex model. (C) 2002 American Institute of Physics.

[DOI: $10.1063 / 1.1497965$ ]

\section{INTRODUCTION}

For more than two decades, the $\mathrm{NH}_{2}$ radical is used as an example to study collision and relaxation processes in small polyatomic molecules which is important for detailed understanding of chemical reactions with particular applications in combustion and atmospheric chemistry. For $\mathrm{NH}_{2}$ in the ground state, $\widetilde{X}^{2} B_{1}$, rotationally inelastic collisions with $\mathrm{He}$ were examined by Dagdigian. ${ }^{1}$ In the excited state, $\widetilde{A}^{2} A_{1}$, efficient quenching by various gases was observed by Halpern et al., ${ }^{2}$ and Donnelly et al. ${ }^{3}$ State-to-state collisional energy transfer (CET) has first been observed by Kroll. ${ }^{4}$ Detailed investigations on rotational energy transfer within $\mathrm{NH}_{2}\left(\widetilde{A}^{2} A_{1}\right)$ originating from collisions with $\mathrm{H}$-atoms were performed by Dixon and Field, ${ }^{5}$ Dearden et al., ${ }^{6}$ and Alwahabi et al., ${ }^{7}$ and with polarization sensitive detection by Truhins et al. ${ }^{8}$ Recently, state-to-state CET in electronically excited $\mathrm{NH}_{2}\left(\widetilde{A}^{2} A_{1}\right)$ with $\mathrm{NH}_{3}$ has been observed by Dong et al. ${ }^{9}$ and by us. ${ }^{10}$ Dong et al. generated $\mathrm{NH}_{2}$ in a combination of a discharge with a supersonic jet and reported populations for collisions at low rotational quantum numbers $N$ $(N<7)$ while we produced $\mathrm{NH}_{2}$ from photodissociation of $\mathrm{NH}_{3}$ and determined state-to-state rate constants for high $(N \geqslant 14)$ rotational quantum numbers. In this very recent work, ${ }^{10}$ we have also given references on state-to-state CET in other polyatomic molecules; further information can be gained from recent reviews by Dagdigian, ${ }^{11}$ Schiffman and Chandler, ${ }^{12}$ and Everitt and De Lucia. ${ }^{13}$

\footnotetext{
a) Author to whom correspondence should be addressed. Electronic mail: jlindne@gwdg.de

${ }^{b)}$ Present address: Laser Zentrum Hannover, Hollerithallee 8, D-30419 Hannover, Germany.
}

Despite the numerous examples, the understanding about collisions of various species with $\mathrm{NH}_{2}$ is far from being complete. A detailed understanding starts to emerge for collisions between $\mathrm{NH}_{2}\left(\widetilde{A}^{2} A_{1}\right)$ and $\mathrm{H}$ atoms, where changes in the amount and orientation of the rotational quantum number are described with the aid of an angular momentum sphere.

In our investigation, state-to-state rate constants for collisions of $\mathrm{NH}_{2}\left(\widetilde{A}^{2} A_{1}\right)$ with $\mathrm{NH}_{3}$ at low rotational quantum numbers are determined. The precursor $\mathrm{NH}_{3}$ is the natural environment, when $\mathrm{NH}_{2}$ is produced by photodissociation, and therefore, collisions between these two species must be understood before studying other systems. For low rotational quantum numbers, the information about energy levels and transition matrix elements is more complete than for the higher ones and changes in the rotational quantum numbers are not always accompanied by vibrational ones. ${ }^{10}$ Therefore, a wider range in rotational quantum numbers of the experimentally determined state-to-state rate constants is expected when compared to our previous work. Such a data set might stimulate theoretical work on this collision process between two polyatomic molecules. In addition, it will then be possible to investigate whether these rate constants can be described by simple scaling laws, one of which has been successfully applied to collisions of $\mathrm{NH}_{2}\left(\widetilde{A}^{2} A_{1}\right)$ with H-atoms, ${ }^{7}$ while others gave an excellent description in a variety of collisions between atoms and diatomic molecules. Although only approximate information about the collision process can be obtained from scaling laws, they are, however, sometimes applied to simulate collision and relaxation processes when state-to-state rate constants can not be measured directly. It is, therefore, instructive to investigate which scaling laws are pertinent. Regarding the interpretation of our results, we will 


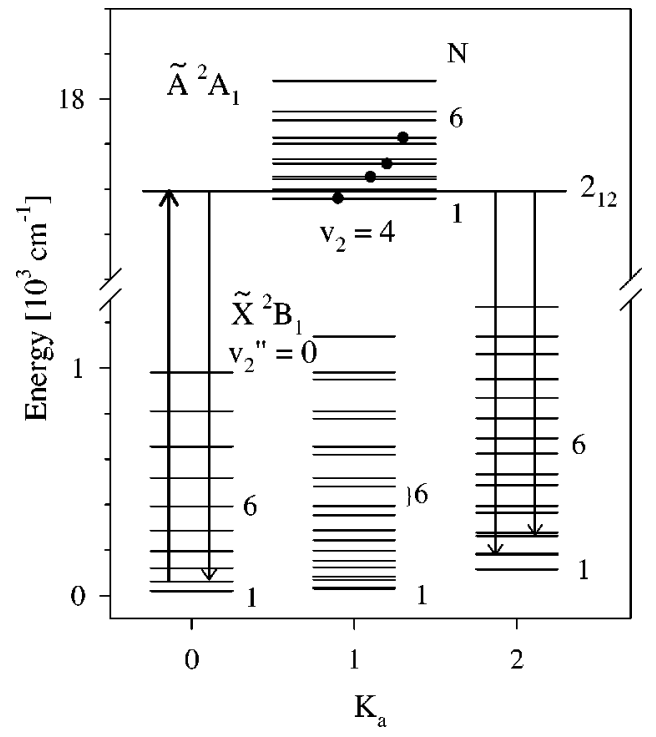

FIG. 1. Energy level diagram of $\mathrm{NH}_{2}$. In the ground (excited) state $\widetilde{X}^{2} B_{1}$ $\left(\widetilde{A}^{2} A_{1}\right)$ rotational levels for $v_{2}^{\prime \prime}=0\left(v_{2}=4\right)$ are shown. Levels populated by CET from $2_{12}$ are labeled with filled circles.

discuss the collision dynamics in a visual picture and with respect to an estimate of the anisotropic part of the interaction potential. Finally we will explore how our results compare with the observations obtained by Dong et al. ${ }^{9}$ under completely different experimental conditions.

As in our previous work, ${ }^{10} \mathrm{NH}_{2}\left(\widetilde{X}^{2} B_{1}\right)$ is produced by $193 \mathrm{~nm}$ photodissociation of $\mathrm{NH}_{3}{ }^{14-17}$ and excited with a pulsed dye laser to the excited state $\widetilde{A}^{2} A_{1}$ as indicated with the upward pointing arrow in the energy level diagram Fig. 1. Upon collisions with $\mathrm{NH}_{3}$, new levels of electronically excited $\mathrm{NH}_{2}\left(\widetilde{A}^{2} A_{1}\right)$ are populated (filled circles in Fig. 1), i.e.,

$$
\begin{aligned}
& \mathrm{NH}_{2}\left(\widetilde{A}^{2} A_{1}, v_{1} v_{2} 0, N_{K_{a}, K_{c}}\right)+\mathrm{NH}_{3} \\
& \quad \rightarrow \mathrm{NH}_{2}\left(\widetilde{A}^{2} A_{1}, v_{1} v_{2} 0, N_{K_{a}^{*}, K_{c}^{*}}^{*}\right)+\mathrm{NH}_{3}^{*},
\end{aligned}
$$

where $v_{1}$ and $v_{2}$ are vibrational quantum numbers in the bent-molecule notation, ${ }^{15} \mathrm{~N}$ is the total angular momentum (without spin) and $K_{a}\left(K_{c}\right)$ are its projections on the axis with the smallest (largest) moment of inertia. The fluorescence of the initially excited level (downward pointing arrows in Fig. 1) and those populated by rotational energy transfer (RET) is observed time and frequency resolved with Fourier transform emission spectroscopy (FTES). The changes of $\mathrm{NH}_{2}\left(\widetilde{A}^{2} A_{1}\right)$ internal energy in this experiment vary from -250 to $+240 \mathrm{~cm}^{-1}$.

\section{RESULTS AND ANALYSIS}

The experimental setup and conditions are identical with those described previously. ${ }^{10,18}$ However, the delay between photodissociation $(193 \mathrm{~nm}, 40 \mathrm{~mJ})$ and excitation pulse is now $10 \mu$ s instead of $20 \mathrm{~ns}$ as it was in our investigation on CET for high rotational quantum numbers. ${ }^{10}$ During this time, the nascent $\mathrm{NH}_{2}\left(\widetilde{X}^{2} B_{1}\right)$ population (preferential in levels with high $\left.N=K_{a}\right)^{15-17}$ following $193 \mathrm{~nm}$ photodissocia-
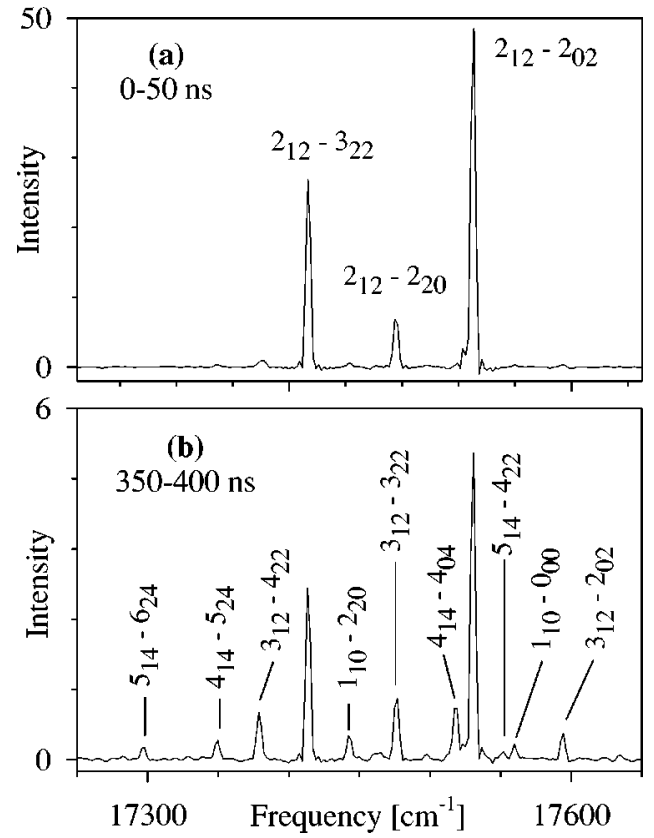

FIG. 2. Dispersed fluorescence observed for (a) $0-50 \mathrm{~ns}$ and (b) 350-400 ns after excitation of the $v_{2}=4-v_{2}^{\prime \prime}=0,2_{12}-2_{02}$ transition of the $\mathrm{NH}_{2}(\widetilde{A}-\widetilde{X})$ system. Transitions originating from the initially excited level are assigned in (a), and collision satellites in (b). The transition $2_{12}-2_{20}$ is overlapped by the collision satellite $3_{12}-3_{22}$.

tion of $\mathrm{NH}_{3}$ relaxes to low rotational quantum numbers. It has been verified with the observation of laser excitation spectra between 17200 and $18200 \mathrm{~cm}^{-1}$ with detection of the total fluorescence and at time delays between 0.5 and 100 $\mu$ s that this time delay is a good compromise between a complete relaxation $(>50 \mu \mathrm{s})$ and a decreasing particle density due to diffusion out of the observation region at longer time delays. However, diffusion within the $0.6 \mu$ s observation time of the time-resolved FTES experiment can be neglected. ${ }^{10}$

\section{A. State-to-state energy transfer and rate constants}

CET in the $\widetilde{A}^{2} A_{1}$ state of $\mathrm{NH}_{2}$ is investigated in $v_{2}=4$ for the rotational levels $2_{12}, 3_{12}, 3_{13}, 4_{13}$, and $4_{14}$ and in $v_{1}=1, v_{2}=2$ for the level $3_{21}$. Typical time-resolved dispersed fluorescence spectra are shown in Fig. 2. In Fig. 2(a), the spectrum is given for the first $50 \mathrm{~ns}$ after the excitation of $\mathrm{NH}_{2}\left(\widetilde{A}^{2} A_{1}, 040,2_{12}\right)$ (compare Fig. 1). Transitions are labeled with quantum numbers assigned from the work of Ross et al. ${ }^{19}$ and Dressler and Ramsay. ${ }^{20}$ The spectrum recorded 350 ns later in Fig. 2(b) shows that additional lines have gained in intensity when compared with the fluorescence from the direct excitation. CET is observed only within the same vibrational state $v_{2}=4$ and for $\Delta K_{a}=0$. Under these conditions, time-dependent relative populations can be obtained after first correcting the spectra for the frequency dependence of the detection system and that of the spontaneous emission. Then the transition intensities are divided by the corresponding Hönl-London factors (see below) and the degeneracy of the upper level. ${ }^{5,21,22}$ For the investigation of RET from the rotational level $2{ }_{12}$ the result of this procedure is shown in Fig. 3. The initially excited level $2{ }_{12}$ decays 


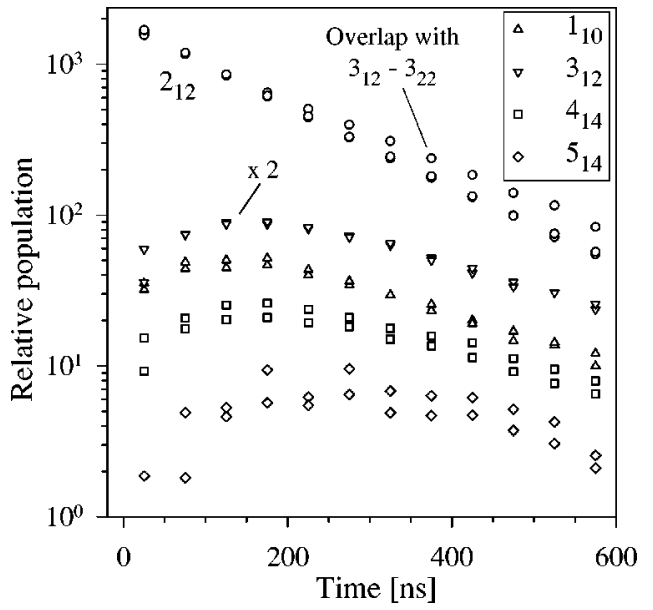

FIG. 3. Time dependent relative populations obtained from the three fluorescence lines of the initially excited level $\widetilde{A}^{2} A_{1}, v_{2}=4,2_{12}$ [closed circles, compare Fig. 2(a)] and two lines from fluorescence of each of the levels (see legend) populated by RET [Fig. 2 (b)]. The relative populations obtained for the level $3_{12}$ have been multiplied by a factor of two for clarity.

exponentially, as can be seen from the figure. From the relative populations calculated from the three fluorescence lines shown in Fig. 2(a), two out of three are almost undistinguishable on top of each other. The relative population obtained from the transition $2_{12}-2_{20}$ differs from that of the other two lines at time delays larger than $200 \mathrm{~ns}$ due to the overlap with the collision satellite $3_{12}-3_{22}$ [compare Fig. 2(a) with 2(b)]. In Fig. 3, the time-dependent populations of the levels $1_{10}$, $3_{12}, 4_{14}$, and $5_{14}$ populated by RET first grow with time and then decay slowly. From the temporal shape of the populations and the single collision conditions in our apparatus, ${ }^{10}$ it can be concluded that all collision satellites originate from primary collisions. Again, Fig. 3 illustrates that the two relative populations obtained from the two fluorescence lines [Fig. 2(b)] for each of the levels $1_{10}, 3_{12}, 4_{14}$, and $5_{14}$ populated by RET are almost the same. For the level $5_{14}$ deviations originate from the small relative populations, i.e., weak intensity of the corresponding transitions. The small difference visible in the populations for the level $4_{14}$ originates from an overlapping line as is verified when $4_{14}$ is the primary excited level.

The Hönl-London factors (HLF) are calculated with a computer program. Eigenvalues and Eigenvectors are obtained from diagonalization of a Hamiltonian matrix which considers centrifugal distortions up to the sixth-order. ${ }^{23}$ For the $\widetilde{A}$ state, rotational constants for a rigid rotator are calculated with the formulas given by Zare (p. 295). ${ }^{24}$ The average $\mathrm{HNH}$ binding angle for $K_{a}=1$ and $v_{2}=4$ and the corresponding bond length are determined from the work of Dixon et al..$^{15}$ and Jungen et al. ${ }^{25}$ For the ground state, rotational constants are taken from Burkholder et al. ${ }^{26}$ HLF are calculated with the formula reported by Dong et al. ${ }^{9,27}$ and do not depend critically on a variation of the $\widetilde{A}$ state binding angle within \pm 5 degrees. As discussed above, the relative populations obtained from the individual fluorescence lines with the same upper level are in good agreement with each other. This demonstrates, that despite Renner-Teller perturbations in the $\widetilde{A}$ state of $\mathrm{NH}_{2},{ }^{15,28}$ the description of the relative line intensities with the calculated HLF is quite accurate. The agreement in our investigation is mostly better than $10 \%$. Finally, our computer program calculates transition frequencies as well when given energies of the $\widetilde{A}$ state are applied. ${ }^{19,20}$

As described before, ${ }^{10}$ state-to-state rate constants are now obtained from the slopes of straight lines fitted to the time-dependent population ratio between collision satellites and the primary excited level (typically 12 data points). ${ }^{29}$ The results are given in Table I together with the corresponding energy gaps. We did not observe any collisions changing the nuclear spin, i.e., transitions from ortho to para $\mathrm{NH}_{2}$ or reverse. In addition, it can be seen from the table that with one exception $\left(4_{13}-1_{11}\right.$, due to overlapping lines), all collisions to final levels energetically below the excited one could be recorded. For CET to those final levels energetically above the excitation whose rotational quantum number $\mathrm{N}$ would be larger by at least one than the last one observed, kinetic energies of at least $300 \mathrm{~cm}^{-1}$ are required. This value is close to the available average kinetic energy in this experiment (see below). Together with a qualitative tendency (verified with the fitting laws applied in Sec. III D) for decreasing state-to-state rate constants with increasing $\Delta N$, this explains why CET to these levels is difficult to observe.

\section{B. Rate constants for total collisional removal}

Radiative lifetimes in the $\widetilde{A}$ state exceed $10 \mu \mathrm{s},{ }^{2,3}$ which is large when compared with collision induced lifetimes of several $100 \mathrm{~ns}$ in our experimental conditions. Therefore, approximate rate constants for total collisional removal can be obtained from the lifetime of the primary excited level (compare Fig. 3). The results are given in the far right column of Table I. Our results are 10\% smaller than those obtained by Halpern et al. ${ }^{2}$ for $K_{a}=0, v_{2}=4$ and about $40 \%$ larger than the rate constants obtained by Donnelly et al. ${ }^{3}$ for $v_{2}=1$ and 2 . Only minor dependences on energy and rovibrational quantum numbers are observed. For each initially excited level, the observed rate for total collisional removal is several times higher than the sum of the state-to-state rate constants for RET within the $\tilde{A}$ state. Therefore, energy transfer out of this level is dominated by electronic quenching and RET is only a minor contribution. The lack of the dependence of the total removal rate constants on the initial level, therefore, refers to electronic quenching.

\section{Identification of the collision partner}

As described in our previous work, ${ }^{10}$ it has been verified that $\mathrm{NH}_{3}$ is the dominant collision partner responsible for the observed collision satellites. As also outlined before, ${ }^{10}$ the gas ensemble contains the photodissociation products $\mathrm{NH}_{2}$, $\mathrm{NH}$, and $\mathrm{H}$ atoms besides the precursor $\mathrm{NH}_{3}$. The concentrations of the three products depend linearly, quadratically or in between on the pulse energy of the dissociation laser, respectively. The particle density of $\mathrm{NH}_{2}(\mathrm{NH})$ is expected and was verified experimentally at pulse energies between 1 and $40 \mathrm{~mJ}$ to depend linearly (quadratically) on the power of the dissociation laser. For the initially excited level $4_{14}$, RET was observed for pulse energies 10 and $40 \mathrm{~mJ}$ of the disso- 
TABLE I. State-to-state and total collisional removal rate constants in $10^{-11} \mathrm{~cm}^{3} /\left(\mathrm{molec}^{*} \mathrm{~s}\right)$ and energy gaps between final level and excitation (in brackets) in $\mathrm{cm}^{-1}$. Errors of the rate constants are in parentheses in units of the last digit. Further rate constants for total collisional removal from levels $5_{15}$ and $8_{18}$ are 76.4(76) and $97.8(98)$ in $10^{-11} \mathrm{~cm}^{3} /\left(\right.$ molec*$\left.^{*} \mathrm{~s}\right)$, respectively.

\begin{tabular}{|c|c|c|c|c|c|c|c|}
\hline \multirow[b]{3}{*}{ Excitation } & \multicolumn{6}{|c|}{$\mathrm{NH}_{2}\left(\widetilde{A}^{2} A_{1}, 040\right)$, ortho nuclear spin symmetry } & \multirow{3}{*}{$\begin{array}{l}\text { Total } \\
\text { removal }\end{array}$} \\
\hline & \multicolumn{6}{|c|}{ Final levels } & \\
\hline & $1_{10}$ & $2_{12}$ & $3_{12}$ & $4_{14}$ & $5_{14}$ & $6_{16}$ & \\
\hline $2_{12}$ & $\begin{array}{c}2.99(29) \\
{[-33]}\end{array}$ & & $\begin{array}{c}7.65(77) \\
{[63]}\end{array}$ & $\begin{array}{c}5.6(11) \\
{[121]}\end{array}$ & $\begin{array}{c}2.68(60) \\
{[237]}\end{array}$ & & $86.9(87)$ \\
\hline $3_{12}$ & $\begin{array}{c}3.39(70) \\
{[-97]}\end{array}$ & $\begin{array}{l}6.1(13) \\
{[-63]}\end{array}$ & & $\begin{array}{c}3.25(65) \\
{[58]}\end{array}$ & $\begin{array}{c}4.72(95) \\
{[174]}\end{array}$ & & $87.5(87)$ \\
\hline $4_{14}, 40 \mathrm{~mJ}$ & $\begin{array}{l}0.65(13) \\
{[-154]}\end{array}$ & $\begin{array}{c}4.14(41) \\
{[-121]}\end{array}$ & $\begin{array}{c}2.63(22) \\
{[-58]}\end{array}$ & & $\begin{array}{c}5.08(34) \\
{[116]}\end{array}$ & $\begin{array}{c}2.7(11) \\
{[193]}\end{array}$ & 76.4(77) \\
\hline $\begin{array}{c}4_{14}, 10 \mathrm{~mJ} \\
7_{16}\end{array}$ & $0.53(14)$ & $4.53(45)$ & $2.54(25)$ & & $\begin{array}{c}4.80(34) \\
3.69(73) \\
{[-254]}\end{array}$ & $\begin{array}{c}1.7(10) \\
5.79^{\mathrm{a}} \\
{[-174]}\end{array}$ & $\begin{array}{l}84.5(84) \\
80.0(80)\end{array}$ \\
\hline
\end{tabular}

\begin{tabular}{|c|c|c|c|c|c|c|}
\hline \multirow[b]{2}{*}{ Excitation } & \multicolumn{5}{|c|}{ Final levels } & \multirow[b]{2}{*}{$\begin{array}{c}\text { Total } \\
\text { removal }\end{array}$} \\
\hline & \multirow{3}{*}{$\begin{array}{c}1_{11} \\
3.41(34) \\
{[-87]}\end{array}$} & $2_{11}$ & $3_{13}$ & $4_{13}$ & $5_{15}$ & \\
\hline $3_{1,3}$ & & $\begin{array}{c}3.47(42) \\
{[-46]}\end{array}$ & & $\begin{array}{c}6.46(64) \\
{[88]}\end{array}$ & $\begin{array}{c}5.50(55) \\
{[156]}\end{array}$ & $80.0(80)$ \\
\hline \multirow[t]{3}{*}{$4_{1,3}$} & & $\begin{array}{l}5.6(26)^{b} \\
{[-134]}\end{array}$ & $\begin{array}{l}5.8(12) \\
{[-88]}\end{array}$ & & $\begin{array}{l}3.90^{\mathrm{a}} \\
{[68]}\end{array}$ & $80.9(81)$ \\
\hline & & \multicolumn{4}{|c|}{$\mathrm{NH}_{2}\left(\widetilde{A}^{2} A_{1}, 120\right)$, ortho nuclear spin symmetry } & \\
\hline & & \multicolumn{3}{|c|}{ Final levels } & & \\
\hline Excitation & & $2_{21}$ & $4_{23}$ & & $5_{23}$ & $\begin{array}{c}\text { Total } \\
\text { removal }\end{array}$ \\
\hline \multirow[t]{2}{*}{$v_{1}=1, v_{2}=2,3_{21}$} & & $4.35(83)$ & $8.2(17)$ & & $6.7(14)$ & $91.9(92)$ \\
\hline & & {$[-54]$} & {$[92]$} & & {$[168]$} & \\
\hline
\end{tabular}

${ }^{a}$ Calculated from scaling law with Eqs. (3) and (4), but in agreement with the observation from an overlapping line from simultaneous excitation of $4_{13}$ and 716 .

${ }^{b}$ Larger error because of overlapping lines.

ciation laser. The resulting state-to-state rate constants are given in Table I for both pulse energies. As can be seen from the table, the results agree within error bars of roughly $10 \%$. The rather large deviation for the rate constants for RET from level $4_{14}$ to $6_{16}$ originates from the poor signal-to-noise $(\mathrm{S} / \mathrm{N})$ ratio in the observation of this transition.

An investigation of the conditions in our experiment further supports $\mathrm{NH}_{3}$ as the dominant collision partner. From known pulse energies of the dissociation laser, the flux in the observation regime is calculated under consideration of reflection and absorption losses of the cylindrical lens and the window of the vacuum apparatus (both made from Quartz) and the absorption of the laser beam by $\mathrm{NH}_{3}$ inside the vacuum chamber. The cross sections for $193 \mathrm{~nm}$ light absorption in $\mathrm{NH}_{3}$ have been reported by Thompson et al. ${ }^{30}$ and Suto and Lee ${ }^{31}$ and the emission profile of an ArF excimer laser by Burnham and Djeu. ${ }^{32}$ The simulation includes focusing of the excimer laser to a stripe of 2 to $3 \mathrm{~mm}$ height, dissociation of $\mathrm{NH}_{3}$ by absorption of laser light in the path between window and observation volume ${ }^{33}$ and diffusion out of the observation region within the $10 \mu$ s between dissocia- tion and excitation laser. It neglects subsequent dissociation of $\mathrm{NH}_{2}$ into $\mathrm{NH}$-molecules and $\mathrm{H}$-atoms. For a dissociation laser pulse energy of $40 \mathrm{~mJ}(10 \mathrm{~mJ})$ the concentration of $\mathrm{NH}_{2}$ is about $12 \%(3 \%)$ of the initial one for $\mathrm{NH}_{3}(\sim 7$ $\times 10^{15} \mathrm{~cm}^{-3}$ ). For $\mathrm{NH}_{2}$ and $\mathrm{NH}$ the influence of diffusion is negligible while for $\mathrm{H}$ atoms it decreases the concentration by a factor of two, i.e., to $6 \%(1.5 \%)$ of the initial one for $\mathrm{NH}_{3}$. The thermalization of the hot $\mathrm{H}$-atoms in $\mathrm{NH}_{3}$ produced by photodissociation of $\mathrm{NH}_{3}{ }^{16,17}$ has been assumed to be similar to that in $\mathrm{N}_{2} \cdot{ }^{34}$ Summarized, the concentration of $\mathrm{NH}_{3}$ in the observation region exceeds that of all other species by at least a factor of 7 .

The experiment and simulation presented above exclude any other dominant collision partner apart from $\mathrm{NH}_{3}$. The state-to-state rate constants for RET out of $4_{14}$ (Table I) leave a possibility for a $10 \%$ increase when the pulse energy of the dissociation laser is quadrupled. This small increase could indicate collisions with $\mathrm{NH}_{2}, \mathrm{NH}$, or $\mathrm{H}$ atoms or originate from the elevated temperature in our experiment, as will be pointed out in the Discussion. 
TABLE II. Parameters obtained from fit of the scaling expression Eq. (3) with $k_{\bar{N}, \bar{K}_{c \rightarrow 0,0}}$ taken from Eqs. (4) (angular momentum power law, AMP) and (5) ( $a$ over $n$ law, AON) and for the fixed vector (FV) model from Eq. (2) only. All models include detailed balance.

\begin{tabular}{ccccc}
\hline \hline & & & & \\
Fit to & $\alpha$, a, or $\eta$ & $\mathrm{k}_{\mathrm{B}} \mathrm{T}\left[\mathrm{cm}^{-1}\right]$ & $\left.\mathrm{k}_{0}^{-11} \mathrm{~cm}^{3}\right]$ & $\sigma$ \\
\hline ECS + AMP, Eqs. (3), (4) & $\alpha=1.35 \pm 0.2$ & $412_{-150}^{+400}$ & $12.3_{-4.0}^{+5.5}$ & 1.40 \\
ECS + AON, Eqs. (3), (5) & $a=8.00_{-2.1}^{+5.0}$ & $445_{-160}^{+500}$ & $0.455_{-0.30}^{+0.55}$ & 1.37 \\
FV, Eq. (2) & $\eta=0.92 \pm 0.2$ & $430^{\mathrm{a}}$ & $26_{-8}^{+18}$ & 1.70 \\
\hline \hline
\end{tabular}

${ }^{\mathrm{a}}$ Fixed, see Sec. II D.

\section{Scaling laws for rotational energy transfer}

To extract information about the collision dynamics, we have applied statistical and dynamical scaling laws to our results (without collisions starting from $v_{1}=1, v_{2}=2,3_{21}$ ). Fitting laws for rotationally inelastic collisions have been reviewed by Brunner and Pritchard. ${ }^{35}$ Models for RET in polyatomic molecules were developed by DePristo et al., ${ }^{36}$ Whitaker and Brechignac, ${ }^{37}$ and Alwahabi et al. ${ }^{7,38}$

Statistical scaling laws, which depend only on the energy difference $\Delta E$ between final and initial level of the collision with no restriction in the change of the magnetic quantum number $M_{N}$ or for $\Delta M_{N}=0$ transitions ${ }^{35}$ result in poor agreement between theory and observation. The normalized standard deviation $\sigma$ is about 3 .

Better agreement is obtained with the fixed vector (FV) model from Alwahabi et al., ${ }^{7}$ which is the first of several modifications proposed by Alwahabi and coworkers. ${ }^{38} \mathrm{Al}-$ though it is the easiest one to apply, the FV model has been proven to be as successful as its derivations, which are expected to be superior only for CET with $\Delta K_{a} \neq 0 .{ }^{38}$ Downward rates are calculated from ${ }^{7}$

$$
k_{N, K_{c} \rightarrow N^{*}, K_{c}^{*}}=k_{0} \exp (-\eta|l|),
$$

where $l$ is the geometrical distance between vectors given by $N_{K_{a}, K_{c}}$ and $N_{K_{a}^{*}, K_{c}^{*}}^{*}$ [Eq. (2) and Fig. 4 in Ref. 7]. $\eta$ and $k_{0}$ are fit parameters. Upward rate constants are calculated from detailed balance [e.g., Eq. (13) in Ref. 5]. The normalized standard deviation $\sigma$ of the fit is about 1.70 and the parameters $k_{0}, \eta$, and $k_{B} T$ are given in Table II. $k_{B}$ is the Boltzmann constant and $T$ the absolute temperature. When $k_{B} T$ is varied in the fit, an unphysical value of $14000 \mathrm{~cm}^{-1}(\sigma$ $=1.43$ ) is obtained, indicating that this fitting law cannot describe the dynamics of the collision process completely. Therefore, $k_{B} T$ has been fixed to the average value which is obtained from the two scaling laws described next.

Good results are obtained with a scaling law for atomsymmetric top collisions proposed by DePristo et al. ${ }^{36}$ From calculated eigenvectors for the rotational wave functions of $\mathrm{NH}_{2}(\widetilde{A})$, and as already indicated by Dagdigian, ${ }^{1} \mathrm{NH}_{2}(\widetilde{A})$ can be approximated as a symmetric top. With no information available about the internal degrees of freedom of the collider $\mathrm{NH}_{3}$ and no obvious indications for resonant energy transfer as in our previous work, ${ }^{10} \mathrm{NH}_{3}$ is treated like the above mentioned atom. State-to-state rate constants from the initially excited level $|e\rangle=\left|\widetilde{A}, 040, N_{1, K_{c}}\right\rangle$ to level $\left|e^{*}\right\rangle$ $=\left|\widetilde{A}, 040, N_{1, K_{c}^{*}}^{*}\right\rangle$ populated by RET can be calculated with a general scaling expression within the energy corrected sudden (ECS) approximation for atom-symmetric top collisions $^{36}$

$$
\begin{aligned}
k_{N, K_{c} \rightarrow N^{*}, K_{c}^{*}}= & \left(2 N^{*}+1\right) \sum_{\bar{N}, \bar{K}}(2 \bar{N}+1) \\
& \times\left(\begin{array}{ccc}
N & N^{*} & \bar{N} \\
-K_{c} & K_{c}^{*} & \bar{K}
\end{array}\right)^{2} A(\tau) k_{\bar{N}, \bar{K} \rightarrow 0,0},
\end{aligned}
$$

where the sum over $\bar{N}$ extends from $\left|N-N^{*}\right|$ to $N+N^{*}$ and $\bar{K}=K_{c}-K_{c}^{*}$. In the present case, the adiabatic factor, $A(\tau),{ }^{36}$ is estimated and verified in fits to be approximately 1, i.e., the collision is approximately sudden, and $k_{\bar{N}, \bar{K} \rightarrow 0,0}$ is described with the angular momentum power (AMP) law ${ }^{35}$

$$
k_{\bar{N} \rightarrow 0}^{\mathrm{AMP}}=k_{0}[\bar{N}(\bar{N}+1)]^{-\alpha},
$$

$k_{\bar{N} \rightarrow 0}^{\mathrm{AMP}}$ does not contain any $K_{c}$ dependence and, therefore, the corresponding index has been dropped. The above formulas are valid only for energetic downward collisions. ${ }^{36}$ Upward collisions are obtained from detailed balance as described above. The values of the parameters $k_{0}, \alpha$, and $k_{B} T$ which fit best to our experimental data are given in Table II. A comparison between observation and fit is shown in Fig. 4 for all
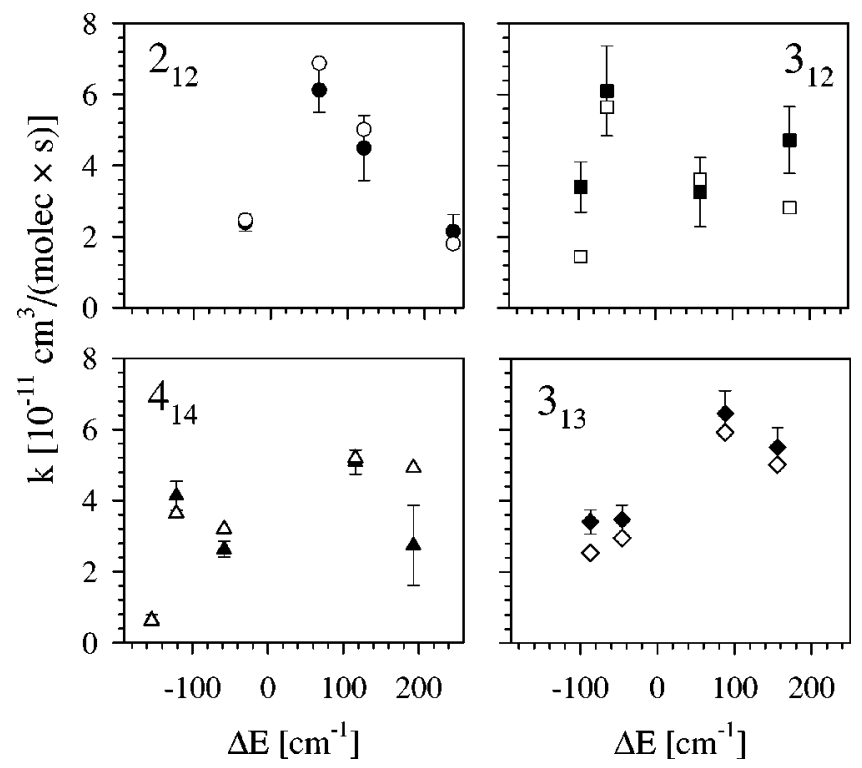

FIG. 4. Comparison of observed (filled symbols with error bars) and fitted (open symbols) state-to-state rate constants for RET from the initially excited levels $2_{12}, 3_{12}, 4_{14}$, and $3_{13}$ in $\widetilde{A}^{2} A_{1}, v_{2}=4$. 
primary excitations with more than three collision satellites. The figure shows clearly that there is no simple dependence of the observed state-to-state rate constants on the energy difference between final and initial level of the collision process. The observations are mostly described within the errors of measurement, as is expected for a standard deviation of $\sigma=1.40$. The considerable error bar in temperature originates because the detailed balance factor $\exp \left(-\Delta E / k_{B} T\right)$ asymptotically approaches 1 with increasing $k_{B} T$, i.e., $k_{B} T$ is calculated from small deviations from 1 of the ratios of stateto-state rate constants for forward and backward collisions. We note that the quality of the fit cannot be increased by multiplying $k_{\bar{N} \rightarrow 0}^{\mathrm{AMP}}$ in Eq. (4) with a factor $\exp (-\beta L(L+1))$ where $L$ is $\bar{N}$ or $\bar{K}$ as suggested by Brunner and Pritchard ${ }^{35}$ or applied by Abel et al. ${ }^{39}$

Fits with about the same standard deviation are also obtained with the so-called AON ( $a$ over $n$ ) fitting law developed by Whitaker and Brechignac. ${ }^{37,40,41}$ In this case, stateto-state rate constants are obtained from Eq. (3) and $k_{\bar{N}, \bar{K} \rightarrow 0,0}$ is replaced with

$$
k_{\bar{N} \rightarrow 0}^{\mathrm{AON}}=k_{0}\left(\frac{a}{n} \ln \left(\frac{a}{n}\right)-\frac{a}{n}+1\right),
$$

where $n=\bar{N} / \lambda$, and $\lambda=1,2, \ldots$ is associated with the $\lambda$ term of the anisotropic part of the Legendre expansion of the interaction potential. ${ }^{42} k_{0}$ and a are fitting parameters and the results are given in Table II for $\lambda=1$ as well. Both parameters are highly correlated and can be varied in the vicinity of the minimum of the standard deviation as long as approximately $a^{*} k_{0}=$ const. All errors in the table correspond to a $68 \%$ confidence interval in the three dimensional (two dimensional for the FV model) parameterspace, i.e., consider variation of the remaining two (one) parameters to minimize chi-square. ${ }^{29,43,44}$ The calculated state-to-state rate constants from the scaling law Eq. (3) in combination with the AON are almost undistinguishable from those calculated with the AMP law. Figure 4, therefore, shows the agreement between observation and fit with the AON law as well.

\section{DISCUSSION}

In this section we shall interpret the results of our fits with respect to the experimental conditions and the dynamics in our collision experiment. Next we shall relate our observations to those obtained from experiments on CET in electronically excited $\mathrm{NH}_{2}$ investigated by Dong et al. ${ }^{9}$ Finally we will discuss the magnitude of the rates for total collisional removal obtained here and in our earlier work on CET in highly rotationally excited $\mathrm{NH}_{2}(\widetilde{A}) .{ }^{10}$

\section{A. Experimental conditions}

A first glance on the fitting parameters given in Table II reveals a surprisingly high temperature $k_{B} T$ around 430 $\mathrm{cm}^{-1}$ in our experiment when compared with the corresponding value for room temperature $\left(k_{B}^{*} 293 \mathrm{~K}\right.$ $\approx 200 \mathrm{~cm}^{-1}$ ). The high temperature is obtained in fits to two models and is in agreement with that determined from detailed balance, $k_{B} T=(540 \pm 200) \mathrm{cm}^{-1}$, of those three state- to-state rate constants with an accuracy better than $20 \%$ where forward and backward collisions are observed. The value of $k_{B} T$ from Table II also fits to the energy dissipation of the excess energy released in the $193 \mathrm{~nm}$ photodissociation of $\mathrm{NH}_{3}$ to the $\mathrm{NH}_{3}$ bath: From the energy of an $193 \mathrm{~nm}$ photon of the dissociation laser and the dissociation energy of $\mathrm{NH}_{3},{ }^{16}$ the excess energy is calculated to $1.78 \mathrm{eV}$. For a slightly different dissociation wavelength, Biesner et al. ${ }^{17}$ reported, that about $20 \%, 54 \%$, and $26 \%$ of the excess energy are released as translational, internal energy in the ground and internal energy in the excited state, respectively. Within the $10 \mu$ s delay between photodissociation of $\mathrm{NH}_{3}$ and excitation of $\mathrm{NH}_{2}$, the translational and excited state energies are dissipated to the $\mathrm{NH}_{3}$ bath. From an analysis of the above mentioned (begin of Sec. II) laser excitation spectra, we estimate that about $2 / 3$ of the ground-state internal energy is transferred to the $\mathrm{NH}_{3}$ bath as well; altogether about $1.4 \mathrm{eV}$ from each $\mathrm{NH}_{2}$ molecule. This energy is distributed among translational and rotational degrees of freedom of the bath molecules. With the above mentioned (Sec. IIC) fraction of $12 \% \mathrm{NH}_{2}$ molecules of the initial $\mathrm{NH}_{3}$ concentration for 40 $\mathrm{mJ}$ pulse energy of the dissociation laser, and a heat capacity of $38 \mathrm{~J} /\left(\mathrm{K}^{*} \mathrm{~mol}\right)$ for $\mathrm{NH}_{3},{ }^{45}$ a temperature increase corresponding to $\Delta k_{B} T=400 \mathrm{~cm}^{-1}$ is obtained. This estimation neglects that part of the $\widetilde{A}$ state internal energy is irradiated as fluorescence and a fraction of hot $\mathrm{H}$ atoms leave the excitation volume by diffusion. Given the considerable error bars for the temperature in our fits (compare Table II) and that obtained from detailed balance, and the remaining uncertainties in the simulation of the conditions in our experiment, the estimated temperature increase of $400 \mathrm{~cm}^{-1}$ is in fair agreement with our observation of $\approx 230 \mathrm{~cm}^{-1}$, i.e., $k_{B} T \approx 430 \mathrm{~cm}^{-1}$. For a dissociation laser pulse energy of 10 $\mathrm{mJ}$, the calculated value for $\Delta k_{B} T$ is about $100 \mathrm{~cm}^{-1}$ and we expect about $60 \mathrm{~cm}^{-1}$ in our experiment, i.e., $k_{B} T$ $\approx 260 \mathrm{~cm}^{-1}$. Because the calculated temperature is higher than the observed one, this might indicate that a smaller fraction of $\mathrm{NH}_{3}$ molecules is photodissociated in the observation region than estimated in Sec. II C.

The elevation of the temperature with pulse energy of the dissociation laser has consequences for the interpretation of the remaining deviations between the state-to-state rate constants for 10 and $40 \mathrm{~mJ}$ given in Table I. Assuming energy independent cross sections, the different temperatures associated with both pulse energies result in an increase of the state-to-state rate constants proportional to the square root of the ratio of the temperatures. ${ }^{46}$ On the other hand, when collisions with other species can be neglected, an increasing laser pulse energy results in a decrease of the $\mathrm{NH}_{3}$ concentration due to a higher fraction of $\mathrm{NH}_{2}, \mathrm{NH}$, and $\mathrm{H}$ and, therefore, lowers the state-to-state rate constants. Altogether an increase by $15 \%$ of the state-to-state rate from 10 to $40 \mathrm{~mJ}$ is expected, which is on the same order as the error bars.

\section{B. Evaluation of the fitting laws}

We now focus on the dynamics of the collision process. We first mention that the conservation of the proton nuclear 
spins restricts the levels in the quantum number $K_{c}^{*}$ which can be populated for a given $N^{*}$ during the collision. For example a collision from an initial level $4_{14}$ in ortho $\mathrm{NH}_{2}$ to a final level $3_{13}$ in para $\mathrm{NH}_{2}$ is theoretically and from other experiments not expected ${ }^{47,48}$ and is not observed here (compare Table II), although energetically possible. Therefore, given the projection $K_{a}$ of the rotational quantum number $N$ on the $a$ axis and either ortho or para $\mathrm{NH}_{2}$, there is a unique relation between $N$ and its projection $K_{c}$ on the $c$ axis.

As outlined above, simple statistically based scaling laws do not give a reasonable description of our observations. This can readily be understood from Fig. 4, which does not show a simple dependence of the state-to-state rate constants on the energy difference as it is typically for the energy or power gap law. ${ }^{35}$ Such behavior has been observed before, e.g., CET of $\mathrm{NH}_{2}(\widetilde{A})$ with $\mathrm{H}$ atoms, ${ }^{7}$ and is discussed for this system by McCaffery et al. ${ }^{49}$ and Alwahabi et al. ${ }^{38}$ As pointed out by McCaffery, ${ }^{49}$ CET is not only controlled by the energy difference between initial and final level but also by the difference in angular momentum. If, as in the present case, exponential or power gap laws fail, the collision dynamics is most likely controlled by the difference in angular momentum as well. This hypothesis is supported by an improved description of our observations with the FV model.

Despite its success in fitting the rates for CET of $\mathrm{NH}_{2}(\widetilde{A})$ with $\mathrm{H}$ atoms, the $\mathrm{FV}$ model lacks a more specific relation to the collision dynamics. This is achieved with the scaling law proposed by DePristo et al. ${ }^{36}$ [Eqs. (3) and (4)] which gives a good fit of our observations. The energy corrected sudden approximation applied in this scaling law allows rotational energies not to be small when compared to translational energies and possible rotation during the collision. It is superior to the infinite order sudden approximation (IOSA), which fails completely in describing our observations and has the above mentioned restrictions. The IOSA only holds for linear molecules and is a limiting form of the scaling relationship derived with the ECS approximation. The ECS approximation relates the state-to-state rate constants between different levels with quantum numbers $N$ and $K_{c}$ to rates $k_{N, K_{c} \rightarrow 0,0}$ for collisions from a rotating molecule to a nonrotating one, compare Eq. (4). Brunner et al..$^{35,50}$ have related the parameters in this angular momentum based power law to the dynamics of the collision process, i.e., to the anisotropic part of the interaction potential. Whitaker and Brechignac accomplished this in a more general approach when developing their AON fitting law [Eq. (5)], ${ }^{37,40}$ and expressed the two fitting parameters $a$ and $k_{0}$ in terms of range and strength of the anisotropic contribution of the interaction potential. It can be shown numerically that in the relevant interval for $N$, the parameters of either law can be adjusted so that the difference between values obtained from Eq. (4) with those from Eq. (5) is small when compared to the absolute value.

Both the AMP and the AON law have been proven to be successful for describing the state-to-state rate constants of various collision processes of atoms with diatomic molecules, ${ }^{37,40,50,51}$ and a collision between two diatomic molecules. ${ }^{41}$ In the derivation of the AON law, Whitaker and Brechignac have assumed that the short-range repulsive part is responsible for the large quantum jumps in $\Delta N,{ }^{37}$ while following Brunner et al. the rotational energy transfer is dominated by the long-range part of the interaction potential only. ${ }^{50}$ In our experiment, we have observed cross sections for RET between 0.5 and $6 \AA^{2}$ with an average value of 3.5 $\AA^{2}$. This value is about half of the hard-sphere collision cross section estimated from typical N-H binding lengths in $\mathrm{NH}_{2}$ and $\mathrm{NH}_{3}$, and $\mathrm{H}$-atom "diameters" and from viscosity measurements ${ }^{45}$ and indicates that the RET is dominated by short-range interactions.

When applying the AON fitting law, it seems surprising that a formalism for an atom-diatom collision works in the present case. In the last section, the average collision energy has been determined to be $430 \mathrm{~cm}^{-1}$. With rotational constants of 9.4 and $6.2 \mathrm{~cm}^{-1},{ }^{28} \mathrm{NH}_{3}$ is populated in a variety of rotational levels and details about the nonspherical structure of the molecule can not be resolved due to averaging. Thus $\mathrm{NH}_{3}$ can be treated as a spherical particle, i.e., like an atom. For $\mathrm{NH}_{2}(\widetilde{A})$, all fits are performed for rotational levels in the vibrational state $v_{2}=4$ and with a projection $K_{a}=1$ of the rotational quantum number on the $a$ axis. As can be seen, for example, from the work of Jungen et al.,${ }^{25}$ the rotational energy corresponding to this $a$ axis rotation alone is $\sim 800$ $\mathrm{cm}^{-1}$. This value is much larger than the above mentioned collision energy. Therefore, the molecule rotates several times around the $a$ axis during the collision process and details of the collision process originating from the bent structure of the $\mathrm{NH}_{2}(\widetilde{A})$ molecule cannot be probed. For the $\mathrm{NH}_{3}$ molecule, therefore, $\mathrm{NH}_{2}(\widetilde{A})$ in $K_{a}=1$ and $v_{2}=4$ appears like a linear molecule. In other words, the description of the collision process regarding the rotation of $\mathrm{NH}_{2}(\widetilde{A})$ is almost in the adiabatic limit for $a$ axis rotation and in the sudden limit for $c$ axis rotation.

\section{Interpretation of the fit parameters}

As mentioned above, the parameters obtained from the fit of our data to Eqs. (3) - (5) contain information about the anisotropic part $V_{\lambda}(r)$ of the Legendre expansion of the interaction potential as a function of intermolecular distance $r$ and angle $\gamma^{42}$

$$
V(r, \gamma)=\sum_{\lambda=0} V_{\lambda}(r) P_{\lambda}(\cos \gamma),
$$

$P_{\lambda}$ is a Legendre polynomial ${ }^{52}$ and usually only terms with low $\lambda$ are considered. $\lambda=0$ belongs to the isotropic part of the interaction potential. Under the assumption that the RET is dominated by one term in Eq. (6), for the AON fitting law, a potential can be calculated from parameters $a$ and $k_{0}, 37,40$

$$
\begin{aligned}
& V_{\lambda}^{\mathrm{AON}}(r)=V_{c} \exp \left(-r / r_{0}\right), r_{0}=\left(\frac{a k_{0}}{2 \bar{u}}\right)^{1 / 2}, \\
& V_{c}=\frac{\hbar \bar{u}}{2 \alpha_{\lambda}}\left(\frac{\bar{u} a}{k_{0}}\right)^{1 / 2},
\end{aligned}
$$

$\bar{u}=\left(8 k_{B} T / \pi \mu\right)^{1 / 2}$ is the mean relative velocity, ${ }^{46} \mu$ the collision reduced mass and $\alpha_{\lambda}$ takes the value 1 for an anisot- 


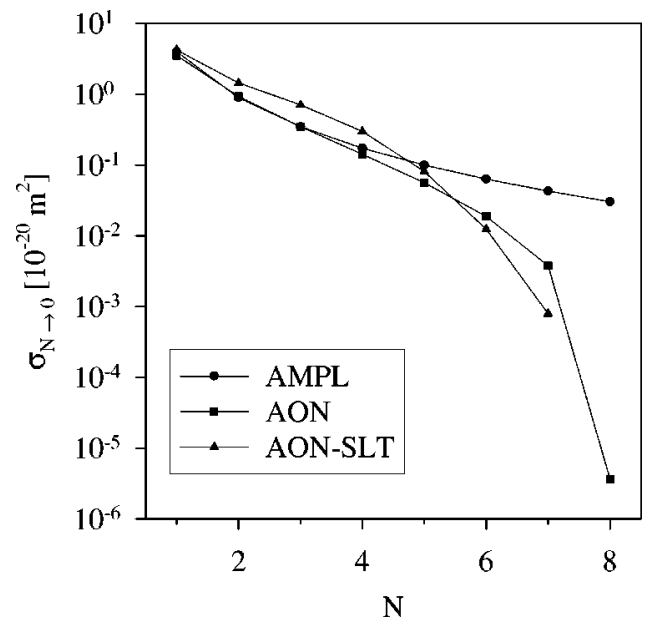

FIG. 5. Cross-sections $\sigma_{N \rightarrow 0}$ obtained from the AMP and AON law and from straight line trajectories (AON-SLT) as described in Sec. III C.

ropy in $P_{1}$ and $3 / 4$ for one in $P_{2} \cdot{ }^{40}$ The exponential form of the anisotropic part of the interaction potential is theoretically reasoned by Roberts $^{53}$ and is also discussed by Margenau and Kestner. ${ }^{54}$

Since $\mathrm{NH}_{2}$ is a heteronuclear molecule we first concentrate on the potential $V_{1}(r)$. From the parameters given in Table II we obtain

$$
r_{0}=1.21 \AA, V_{c}=151 \mathrm{~cm}^{-1} .
$$

The error bars of $a$ and $k_{0}$ result in about $10 \%$ uncertainty of $r_{0}$, while $V_{c}$ is determined within a factor of 2 only. To illuminate the underlying collision dynamics and to check the consistency of the applied model, we calculated the probability function $P_{N \rightarrow N^{*}}(b)$ as a function of the impact parameter $b$ using straight line trajectories (SLT). ${ }^{42,46}$ $P_{N \rightarrow N^{*}}(b)$ can be obtained from the following equation: ${ }^{37,40,42,55}$

$$
\begin{aligned}
& P_{N \rightarrow N^{*}}(b)=\left(2 N^{*}+1\right)\left(J_{\left|N-N^{*}\right| / \lambda}\left(q_{\lambda}\right)\right)^{2}, \\
& q_{\lambda}=\frac{\alpha_{\lambda}}{\hbar} \int_{-\infty}^{\infty} V_{\lambda}[R(t)] d t,
\end{aligned}
$$

$J_{m}$ is a Bessel function of the order $m .^{52}$ The cross-section $\sigma_{N \rightarrow 0}$ is given by $36,37,40,42,55$

$$
\sigma_{N \rightarrow 0}=2 \pi \int_{0}^{\infty} P_{N \rightarrow 0}(b) b d b,
$$

and is related to the observed rates $k_{N \rightarrow 0}$ via $k_{N \rightarrow 0}$ $=\sigma_{N \rightarrow 0} \bar{u}^{40,46}$ The above formulas require the collisions to be sudden (as verified in Sec. II D) and the transferred energy to be small when compared with the collision energy, i.e., $k_{B} T$. Both conditions are roughly true for most of the observed collisions (see Table I). In addition, as pointed out above, the AON fitting law assumes that the collision is dominated by short-range interactions which cutoff at impact parameters $b_{\max }=r_{0} \ln (a / n)$, i.e., the upper limit in the integral of Eq. (10) is replaced by this N-dependent value. ${ }^{37,40}$ The collision cross sections obtained from the AMP and the AON law and the cross section calculated from Eqs. (7) to (10), denoted as AON-SLT, are displayed in Fig. 5. Given several approximations in the derivation of the AON fitting law, there is reasonable consistency between the input $(\mathrm{AON})$ and the calculated cross section (AON-SLT).

It can be seen from Fig. 5 that the cross-sections $\sigma_{N \rightarrow 0}$ obtained from the AON and AMP fitting laws are similar for $\mathrm{N}$ between 1 and 5 , but differ significantly for $N \geqslant 6$. However, for $N \geqslant 6$, the cross sections are already more than two orders of magnitude smaller than for $N=1$ and, therefore, have only minor influence on the state-to-state rate constants calculated with the ECS approximation Eq. (3). In this context, it should be noted that the weighting factors, i.e., the product of the degeneracy factor with the square of the three-J symbol in the sum in Eq. (3), are generally largest for rotational quantum numbers $\bar{N}$ between $N$ and $N^{*}$. Therefore the state-to-state rate constants are mainly determined through those $k_{N \rightarrow 0}=\bar{u} \quad \sigma_{N \rightarrow 0}$ with a small value $N$. This also explains why the introduction of the factor $\exp (-\beta \bar{N}(\bar{N}+1))$ in Eq. (4) (see Sec. II D) does not improve the quality of our fits.

A look on the probability function $P_{N \rightarrow N^{*}}(b)$ shows, that collisions with $\Delta N=1$ are most likely expected for impact parameters $b$ of $2 \AA$, while the largest observed change in the rotational quantum number, $\Delta N=3$, corresponds to $b=1 \AA$. As an example, this would be the case when the center-of-mass of the $\mathrm{NH}_{3}$ molecule hits one of the H-atoms of $\mathrm{NH}_{2}\left(\widetilde{A}^{2} A_{1}\right)$ and the corresponding $\mathrm{N}-\mathrm{H}$ bonding $(\sim 1.0$ $\AA)^{15,25,45}$ is almost perpendicular to the trajectory of the approaching $\mathrm{NH}_{3}$ molecule. We note that within the error of $V_{c}$, these values could be up to $1 \AA$ larger and RET with $\Delta N=5$ would have its maximum probability for $b=1.3 \AA$.

Finally, in this section we illuminate whether it is justified to limit the anisotropic part of the interaction potential to one term in the expansion in Eq. (6). As discussed in Sec. III B, $\mathrm{NH}_{2}(\widetilde{A})$ could be considered as a heads-tail symmetric linear molecule for the collision process and the interaction potential Eq. (6) might, therefore, contain a term corresponding to $\lambda=2$. CET originating from this part of the interaction potential would only effect collisions with even $\Delta N{ }^{35,42}$ We have, therefore, fitted from the state-to-state rate constants given in Table I those with even $\Delta N$ and odd $\Delta N$ separately with the AON and the AMP in combination with the ECS approximation. We have also performed a combined fit of these data with the AON + ECS fitting law, where RET with all $\Delta N$ is described with parameters $a_{1}$ and $k_{01}$ as in Eq. (5), and in addition, RET for even $\Delta N$ with parameters $a_{2}$ and $k_{02}$. As described in Sec. II D, only energetic downward collisions are calculated, and energetic upward collisions are obtained from detailed balance with a fit parameter $k_{B} T$. However, none of the fits resulted in an improved description of our data. We, therefore, conclude that the anisotropic part of the interaction potential for collisions between $\mathrm{NH}_{2}(\widetilde{A})$ and $\mathrm{NH}_{3}$ is dominated by the first term in the Legendre expansion Eq. (6), i.e., $V_{1}(r)$.

\section{Comparison with investigations in beam-gas conditions}

So far, two investigations have been performed on stateto-state energy transfer in electronically excited $\mathrm{NH}_{2}$ with $\mathrm{NH}_{3} \cdot{ }^{9,10}$ The work of Dong et al. is closely related to this 
one with respect to the rovibrational levels in which CET transfer is observed, but differs strongly in its experimental realization.

Dong et al. ${ }^{9}$ have given relative populations. Because the authors claim that their investigation is in the single collision limit as well, we have converted relative populations to relative rate constants by dividing the final populations by the initial ones. ${ }^{10}$ As compared to Dong and co-workers, we did not observe CET for $\Delta K_{a} \neq 0$. Therefore, only RET within $K_{a}=1, v_{2}=4$ is compared with an equivalent subset of the data set of Dong (same relative errors as in Table I), and all values obtained by Dong et al. in $v_{2}=4, K_{a}=1(20 \%$ relative error). When fitted with the FV model, for the equivalent data set of Dong et al., the standard deviation is 1.4 and all values in $K_{a}=1, v_{2}=4$ can be described with $\sigma=2.2$. The coefficients $\eta$ distinguish with values of $0.53_{-0.15}^{+0.20}$ and $0.35 \pm 0.07$ significantly from our result (compare Table II). Fitted temperatures $k_{B} T$ are about 100 $\pm 10 \mathrm{~cm}^{-1}$. The quality of these fits is better than for those performed with the AMP law in combination with the ECS approximation. The observations of the equivalent data subset of Dong et al. can be fitted with Eqs. (3) and (4) only with a standard deviation of 2.2 with $\alpha=0.92 \pm 0.30$ and $k_{B} T=86 \pm 10 \mathrm{~cm}^{-1}$, while $\alpha=0.61 \pm 0.30$ and $k_{B} T=105$ $\pm 6 \mathrm{~cm}^{-1}, \sigma=2.4$, are obtained for all observations in $K_{a}$ $=1$ and $v_{2}=4$. We note here that Dong et al. could not fit their data satisfactorily with the turning point model. ${ }^{38}$

From the fitted temperatures in this experiment, it seems that CET is not due to translational to rotational collisions between $\mathrm{NH}_{2}(\widetilde{A})$ and the remnant $\mathrm{NH}_{3}$ in the vacuum chamber. In this case a translational energy of $360 \mathrm{~cm}^{-1}$ is expected $^{9}$ and estimated from the conditions in this experiment. ${ }^{56}$ However, such an experimental realization requires distances of at least $5 \mathrm{~cm}$ between nozzle and observation zone, where the beam would be disturbed due to collisions with the remaining gas $(\sim 4 \mathrm{~Pa})$ in the main chamber. Below this distance, collisions within the molecular beam are likely to dominate the collision process and low temperatures, as obtained from the above mentioned fits, are expected. ${ }^{56}$

When compared to our work, a further difference originates from the observation of CET with $\Delta K_{a}= \pm 2$ through Dong et al. ${ }^{9}$ which in the bent molecule notation are always related to a change of the vibrational quantum number $v_{2}$ by $\Delta v_{2}=\mp 1 \cdot{ }^{15,25}$ Based on calculated HLF as described in Sec. II A, transition moments ${ }^{25}$ which relate the intensity of the fluorescence from levels with $K_{a}=1$ to that with $K_{a}=3$, and populations given by Dong et al. (Table II), ${ }^{9}$ we conclude that we should be able to detect these collisions within our $\mathrm{S} / \mathrm{N}$ ratio as well. However, no such collisions could be observed. We attribute this lack to the difference in both experiments. Besides those outlined in the previous paragraph, the five times excess of Ar concentration over that of $\mathrm{NH}_{3}$ in Dong's experiment might be an explanation.

\section{E. Rate constants for total collisional removal}

Rate constants for total collisional removal observed in the present investigation and earlier ${ }^{10}$ are about
$80 \times 10^{-11} \mathrm{~cm}^{3} /($ molec s). Roughly a fraction of 20 $\times 10^{-11} \mathrm{~cm}^{3} /($ molec s) originates from CET within the excited $\widetilde{A}^{2} A_{1}$ state of $\mathrm{NH}_{2}$. Therefore, the rate constant for electronic quenching is about $60 \times 10^{-11} \mathrm{~cm}^{3} /($ molec s). As can be seen in Table I and as discussed in Sec. II A, the influence of the pulse energy of the dissociation laser on the total removal rate constant is small. The above given rate for electronic quenching is, therefore, close to that at room temperature which is assumed for conversion of cross sections into rate constants within this section.

Models to describe electronic quenching have been proposed by several authors and the earlier work is reviewed by Yardley. ${ }^{46}$ Here we focus on an approach presented by Holtermann et al. ${ }^{57}$ which is based on the collision complex model and allows the determination of an upper limit for absolute cross sections. According to this model, the electronic energy of $\mathrm{NH}_{2}$ in the $\widetilde{A}^{2} A_{1}$ state is partitioned over a $\mathrm{NH}_{2}-\mathrm{NH}_{3}$ complex, which is formed through the attractive long-range interactions dipole-dipole, dipole-quadrupole, dipole-induced dipole and dispersion. ${ }^{58,59}$ For these interactions, Hoyermann et al. have given the corresponding contributions to the total cross section for the complex formation as a function of dipole and quadrupole moments, polarizability and ionization potential of the collision partners. ${ }^{57}$ The probability for a nonradiative decay of the complex depends on the intramolecular dynamics, which are not known. However, an upper limit of the cross section for electronic quenching can be calculated, if this probability is assumed to be one.

For $\mathrm{NH}_{3}$, dipole moment and polarizability are obtained from Thayer and Yardley ${ }^{60}$ and the quadrupole moment from Kukolich. ${ }^{61}$ For $\mathrm{NH}_{2}$, to our knowledge, none of the mentioned values has been measured. However, the dipole moment in the electronic $\widetilde{A}$ state has been calculated by Brown and Williams, ${ }^{62}$ and Bell and Schaefer III, ${ }^{63}$ to almost identical values of 0.82 Debye. We assume the polarizability to be similar to that of $\mathrm{NH}_{3}$ and the quadrupole moment to be 0 . The ionization potentials of both molecules are taken from Radzig and Smirnov. ${ }^{64}$ With the formulas (in cgs units) given by Holtermann et al. and under consideration of thermal averaging of the dipole orientations, as described by the authors, ${ }^{57}$ a cross section for electronic quenching of $170 \AA^{2}$, corresponding to a rate constant of 145 $\times 10^{-11} \mathrm{~cm}^{3} /($ molec s) is obtained. This value is about a factor 2.4 larger than the observed one. For the other colliders, for which rate constants for total collisional removal (assumed to be dominated by electronic quenching) have been measured by Halpern et al. ${ }^{2}$ the ratios between the calculated (apply Refs. 57, 60, and 64) and the observed ones are between 1.6 and 3. Given the rough estimations outlined above and that the model calculates only upper values of the cross sections for electronic quenching, a fair agreement with observation is obtained. The deviation can be explained if less than half of the $\mathrm{NH}_{2}(\widetilde{A})-\mathrm{NH}_{3}$ complexes decay nonradiative. 


\section{CONCLUSIONS}

In this work, state-to-state rate constants for RET within $\mathrm{NH}_{2}\left(\tilde{A}^{2} A_{1}\right), v_{2}=4, K_{a}=1$ originating from collisions with $\mathrm{NH}_{3}$ have been determined. $\mathrm{NH}_{2}$ is obtained from photodissociation of $\mathrm{NH}_{3}$. Although two other additional species are produced in this process, it is an easier experiment to control than the generation of $\mathrm{NH}_{2}$ in discharges, where rare gases and possibly other ingredients contribute to the variety of possible collision partners. ${ }^{5,6,9}$ The introduction of a delay between the photolysis of $\mathrm{NH}_{3}$ and the excitation of $\mathrm{NH}_{2}$ allows one to access low rotational quantum numbers as well as highly rotationally excited levels, which were investigated previously. ${ }^{10}$ In principle, it seems possible, although very tedious, to explore the relaxation of highly rotationally excited $\mathrm{NH}_{2}$ in its electronic ground state by recording various laser excitation spectra as a function of the delay.

The observed state-to-state rate constants for RET in the $\widetilde{A}$ state can reasonably well be described with the AMP and AON fitting laws in combination with the ECS approximation. Following the interpretation provided with the AON fitting law, the collision is dominated by repulsive shortrange forces of the anisotropic part of the interaction potential. The most probable impact parameters are in accordance with the classical picture where $\mathrm{NH}_{3}$ directly hits $\mathrm{NH}_{2}$. RET with $\Delta N=1$ occurs at impact parameters where both molecules "touch each other." RET with $\Delta N=3$ might be associated with a picture where the center-of-mass of the $\mathrm{NH}_{3}$ molecule hits the $\mathrm{H}$-atom of $\mathrm{NH}_{2}$ with the $\mathrm{N}-\mathrm{H}$ bonding perpendicular to the velocity vector of the approaching $\mathrm{NH}_{3}$. Higher changes in the rotational quantum number would be attributed to smaller impact parameters. This model should not be stressed too much, but it visualizes our observations.

The present investigation can only cover a small part of possible experiments on the $\mathrm{NH}_{2}\left(\widetilde{A}^{2} A_{1}\right)-\mathrm{NH}_{3}$ collision system. First, different vibrational states and other quantum numbers $K_{a}$ can be selected to study the influence on the collision process. Dependencies are expected, because the state-to-state rate constants measured for RET from the initial level $v_{1}=1, v_{2}=2,3_{21}$ (compare Table I) cannot be sucessfully included in our fits. An investigation of $K_{a}=0$ might be appealing, especially to investigate the influence of the barrier in the $\widetilde{A}$ state potential of $\mathrm{NH}_{2}$ on the collision process. A variation of the vibrational quantum numbers is required to probe RET from levels below and above the barrier. However, when compared to the present conditions, the available pulse energy of the exciting dye laser in our experiment is significantly reduced in the spectral region required for exciting the corresponding levels. This would mean 10 times increased averaging time for the time-resolved spectra.

The discussion of the rates for electronic quenching of $\mathrm{NH}_{2}$ in its $\widetilde{A}$ state in Sec. III E indicates that the collision complex model is a reasonable approach. It also shows the interplay between investigations of CET and determination of dipole and quadrupole moments. More experimental information on these molecular properties needs to be extracted. In the $\widetilde{A}$ state potential for $K_{a}=0, \mathrm{NH}_{2}$ provides the possibility to switch between levels with vanishing and nonvanishing dipole moment by exciting above and below the barrier. By observing the electronic quenching of these levels, the contribution of specific interactions could be studied.

\section{ACKNOWLEDGMENTS}

The authors thank the Deutsche Forschungsgemeinschaft (DFG) for financially supporting this work. J.L. thanks the DFG for a Habilitationsstipendium and R.W. the Graduiertenkolleg "Photonen, atomare Teilchen und deren Wechselwirkungen" for support in the final stage of his work. Helpful discussions with B. Abel, J. Jethwa, and A.-N. Unterreiner in the final stage of this manuscript are greatfully acknowledged.

${ }^{1}$ P. J. Dagdigian, J. Chem. Phys. 90, 2617 (1989).

${ }^{2}$ J. B. Halpern, G. Hancock, M. Lenzi, and K. H. Welge, J. Chem. Phys. 63, 4808 (1975).

${ }^{3}$ V. M. Donnelly, A. P. Baronavski, and J. R. McDonald, Chem. Phys. 43, 283 (1979).

${ }^{4}$ M. Kroll, J. Chem. Phys. 63, 319 (1975).

${ }^{5}$ R. N. Dixon and D. Field, Proc. R. Soc. London, Ser. A 366, 247 (1979).

${ }^{6}$ S. J. Dearden, R. N. Dixon, and D. Field, J. Chem. Soc., Faraday Trans. 2 78, 1423 (1982).

${ }^{7}$ Z. T. Alwahabi, C. G. Harkin, A. J. McCaffery, and B. J. Whitaker, J. Chem. Soc., Faraday Trans. 2 85, 1003 (1989).

${ }^{8}$ K. Truhins, Z. T. Alwahabi, M. Auzinsh, A. J. McCaffery, and Z. Rawi, J. Chem. Phys. 106, 3477 (1997); K. Truhins, A. J. McCaffery, Z. T. Alwahabi, and Z. Rawi, ibid. 107, 733 (1997).

${ }^{9}$ F. Dong, X. Li, M. Zhang, X. Wang, and N. Lou, J. Chem. Phys. 111, 10578 (1999).

${ }^{10}$ R. Wilhelm and J. Lindner, J. Chem. Phys. 114, 7379 (2001).

${ }^{11}$ P. J. Dagdigian, in The Chemical Dynamics and Kinetics of Small Radicals, edited by K. Liu and A. Wagner (World Scientific, Singapore, 1995), Part I, p. 315.

${ }^{12}$ A. Schiffman and D. W. Chandler, Int. Rev. Phys. Chem. 14, 371 (1995).

${ }^{13}$ H. O. Everitt and F. C. De Lucia, in Advances in Atomic, Molecular, and Optical Physics, Vol. 35 (Academic, San Diego, 1995).

${ }^{14}$ V. M. Donnelly, A. P. Baronavski, and J. R. McDonald, Chem. Phys. 43, 271 (1979).

${ }^{15}$ R. N. Dixon, S. J. Irving, J. R. Nightingale, and M. Vervloet, J. Chem. Soc., Faraday Trans. 87, 2121 (1991).

${ }^{16}$ J. Biesner, L. Schnieder, J. Schmeer, G. Ahlers, X. Xie, K. H. Welge, M. N. R. Ashfold, and R. N. Dixon, J. Chem. Phys. 88, 3607 (1988).

${ }^{17}$ J. Biesner, L. Schnieder, G. Ahlers, X. Xie, K. H. Welge, M. N. R. Ashfold, and R. N. Dixon, J. Chem. Phys. 91, 2901 (1989).

${ }^{18}$ J. Lindner, O. Stahlhut, R. Wilhelm, and K. Ermisch, Rev. Sci. Instrum. 69, 1629 (1998).

${ }^{19}$ S. C. Ross, F. W. Birss, M. Vervloet, and D. A. Ramsay, J. Mol. Spectrosc. 129, 436 (1988)

${ }^{20}$ K. Dressler and D. A. Ramsay, Philos. Trans. R. Soc. London, Ser. A 251, 553 (1959).

${ }^{21}$ W. Bingel, Theorie der Molekülspektren (Verlag Chemie, Weinheim, 1967).

${ }^{22}$ E. E. Whiting, A. Schadee, J. B. Tatum, J. T. Hougen, and R. W. Nicholls, J. Mol. Spectrosc. 80, 249 (1980).

${ }^{23}$ W. Gordy, and R. L. Cook, Microwave Molecular Spectra, 3rd ed., in Techniques of Chemistry (Wiley, New York, 1984), Vol. XVIII.

${ }^{24}$ R. N. Zare, Angular Momentum (Wiley, New York, 1988).

${ }^{25}$ Ch. Jungen, K-E. J. Hallin, and A. J. Merer, Mol. Phys. 40, 25 (1980).

${ }^{26}$ J. B. Burkholder, C. J. Howard, and A. R. W. McKellar, J. Mol. Spectrosc. 127, 415 (1988).

${ }^{27}$ This equation can be calculated with formulae given by Zare, Ref. 24. Insert in Eq. (6.116) the wave functions (6.120) and (6.121) and simplify the expression with (3.114) and (2.28). The dipole moment is given by Eq. (5.4) under consideration of Table 6.2 ( $I^{r}$ representation), and for $\mathrm{NH}_{2}$ only $\mu_{c} \neq 0$. Application of (2.32) gives the result.

${ }^{28} \mathrm{G}$. Herzberg, Molecular spectra and molecular structure (van Nostrand Reinhold, New York, 1966), Vol. III.

${ }^{29}$ P. R. Bevington, Data Reduction and Error Analysis for the Physical Sciences (McGraw-Hill, New York, 1969). 
${ }^{30}$ B. A. Thompson, P. Harteck, and R. R. Reeves, Jr., J. Geophys. Res. 68, 6431 (1963).

${ }^{31}$ M. Suto and L. C. Lee, J. Chem. Phys. 78, 4515 (1983).

${ }^{32}$ R. Burnham and N. Djeu, Appl. Phys. Lett. 29, 707 (1976).

${ }^{33}$ The idea is that the leading edge of the $193 \mathrm{~nm}$ laser pulse reduces the absorption for the tailing edge because the particle density of $\mathrm{NH}_{3}$ is reduced by photodissociation. This increases the flux in the observation region. We have accounted for this effect by dividing the laser pulse in 100 slices so that Beer's law is a good approximation for each slice.

${ }^{34}$ J. Park, N. Shafer, and R. Bersohn, J. Chem. Phys. 91, 7861 (1989).

${ }^{35}$ T. A. Brunner and D. Pritchard, in Advances in Chemical Physics, edited by K. P. Lawley (Wiley, Chichester 1982), Vol. L.

${ }^{36}$ A. E. DePristo, S. D. Augustin, R. Ramaswamy, and H. Rabitz, J. Chem. Phys. 71, 850 (1979).

${ }^{37}$ B. J. Whitaker and P. Brechignac, Chem. Phys. Lett. 95, 407 (1983).

${ }^{38}$ Z. T. Alwahabi, N. A. Besley, A. J. McCaffery, M. A. Osborne, and Z. Rawi, J. Chem. Phys. 102, 7945 (1995).

${ }^{39}$ B. Abel, N. Lange, F. Reiche, and J. Troe, J. Chem. Phys. 110, 1389 (1999).

${ }^{40}$ P. Brechignac and B. J. Whitaker, Chem. Phys. 88, 425 (1984).

${ }^{41}$ P. Brechignac and B. J. Whitaker, J. Chem. Phys. 84, 2101 (1986)

${ }^{42}$ R. D. Levine and R. B. Bernstein, Molecular Reaction Dynamics and Chemical Reactivity (Oxford University Press, Oxford, 1987).

${ }^{43}$ W. H. Press, B. P. Flannery, S. A. Teukolsky, and W. T. Vetterling, Numerical Recipes (Cambridge University Press, Cambridge, 1989).

${ }^{44}$ M. Lampton, B. Margon, and S. Bowyer, Astrophys. J. 208, 177 (1976).

${ }^{45}$ CRC Handbook of Chemistry and Physics, edited by D. R. Lide, 75th edition (CRC Press, Boca Raton, 1994).

${ }^{46} \mathrm{~J}$. T. Yardley, Introduction to Molecular Energy Transfer (Academic, New York, 1980).

${ }^{47}$ K. F. Bonhoeffer and P. Harteck, Zeitschr. f. physikal. Chemie B4, 113 (1929); E. Wigner, Zeitschr. f. physikal. Chemie B23, 28 (1933).
${ }^{48}$ B. Abel, S. L. Coy, J. J. Klaassen, and J. I. Steinfeld, J. Chem. Phys. 96, 8236 (1992).

${ }^{49}$ A. J. McCaffery, Z. T. Alwahabi, M. A. Osborne, and C. J. Williams, J. Chem. Phys. 98, 4586 (1993).

${ }^{50}$ T. A. Brunner, T. P. Scott, and D. E. Pritchard, J. Chem. Phys. 76, 5641 (1982).

${ }^{51}$ T. A. Brunner, R. D. Driver, N. Smith, and D. E. Pritchard, J. Chem. Phys. 70, 4155 (1979); T. A. Brunner, N. Smith, and D. E. Pritchard, Chem. Phys. Lett. 71, 358 (1980); T. A. Brunner, N. Smith, A. W. Karp, and D. E. Pritchard, J. Chem. Phys. 74, 3324 (1981).

${ }^{52}$ Handbook of Mathematic Functions, edited by M. Abramowitz and I. A. Stegun (Dover, New York, 1968).

${ }^{53}$ C. S. Roberts, Phys. Rev. 131, 203 (1963).

${ }^{54} \mathrm{H}$. Margenau and N. R. Kestner, Theory of Intermolecular Forces, 2nd ed. (Pergamon, New York, 1971).

${ }^{55}$ C. Nyeland and G. D. Billing, Chem. Phys. 40, 103 (1979); 60, 359 (1981).

${ }^{56}$ D. R. Miller, in Atomic and Molecular Beam Methods, edited by G. Scoles (Oxford University Press, Oxford, 1988), Vol. 1.

${ }^{57}$ D. L. Holtermann, E. K. C. Lee, and R. Nanes, J. Chem. Phys. 77, 5327 (1982).

${ }^{58}$ P. W. Atkins, Physical Chemistry, 5th ed. (Oxford University Press, Oxford, 1997).

${ }^{59}$ J. O. Hirschfelder, C. F. Curtis, and R. B. Bird, Molecular Theory of Gases and Liquids, 2nd ed. (Wiley, New York, 1964).

${ }^{60}$ C. A. Thayer and J. T. Yardley, J. Chem. Phys. 57, 3992 (1972).

${ }^{61}$ S. G. Kukolich, Chem. Phys. Lett. 12, 216 (1971); 7, 401 (1970).

${ }^{62}$ R. D. Brown and G. R. Williams, Mol. Phys. 25, 673 (1973).

${ }^{63}$ S. Bell and H. F. Schaefer III, J. Chem. Phys. 67, 5173 (1977).

${ }^{64}$ A. A. Radzig and B. M. Smirnov, Reference Data on Atoms, Molecules and Ions (Springer, Berlin, 1985). 